\title{
TIEing down vascular integrity during sepsis
}

Sepsis is a severe systemic disease, triggered by pathogen invasion of the bloodstream, for which treatment is limited to general supportive care and mortality rates are high. Recently, a study in mice found that unusual activity by an angiopoietin 2 (ANG2)targeted antibody preserves vascular endothelial integrity during sepsis through activation of tyrosine-protein kinase receptor TIE2 as well as improving survival. This work could provide the foundation for a muchneeded specific therapy for sepsis.

Vascular inflammation and breakdown of the endothelium compromise vascular integrity during sepsis. TIE2 signalling on endothelial cells stabilizes vascular barrier function, suggesting a potential therapeutic target. However, approaches using analogues or mimetics of the

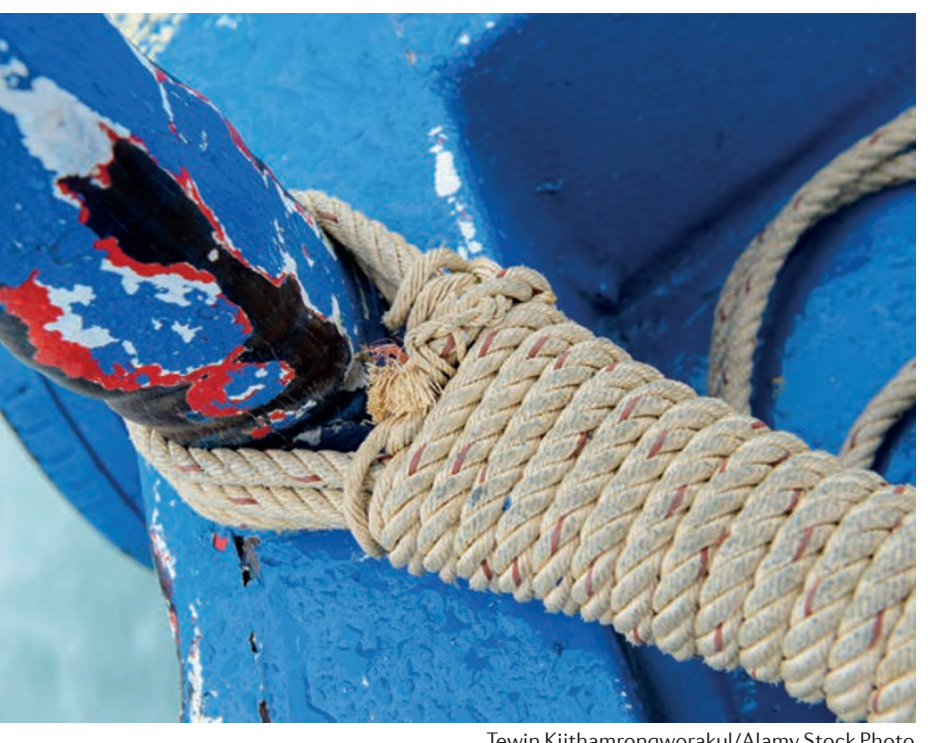

endogenous TIE2 ligand, ANG1, have been limited by the short half-life and nonspecific binding of these drugs.

For the current study, Han et al. developed a human monoclonal antibody that targets the TIE2 antagonist ANG2, which they termed ABTAA. This antibody displayed unusual activity in human umbilical vein endothelial cells in culture: in the presence of ANG2, addition of ABTAA stimulated phosphorylation and downstream signalling of TIE2.

As such, the antibody had converted a TIE2 antagonist into a TIE2 agonist.

Biochemical analysis indicated that the antibody forms an ABTAA-ANG2 oligomerized complex in a 1:2 ratio. The authors suggest that the conformation adopted by ABTAA-bound ANG2 enabled the complex to act as a novel TIE2 agonist.

Next, the authors tested ABTAA in a severe sepsis model in mice involving caecal ligation and puncture (CLP). To mimic a clinically relevant postinfection-treatment setting, mice were intravenously injected with antibody at 6 and 18 hours after CLP. Compared with control mice, most of which died within 96 hours, $40 \%$ of mice treated with ABTAA remained alive at 1 week. Moreover, combination of ABTAA and antibiotics boosted survival at 1 week from $20 \%$ (with antibiotics alone) to $70 \%$. Similar results were observed in two other mouse models of sepsis - endotoxaemia and bacteraemia - suggesting that ABTAA could be broadly effective in sepsis from various causes.
In mice genetically deficient in Ang2 or Tie2 that underwent CLP, ABTAA did not improve survival rates, demonstrating the necessity for both ANG2 and TIE2 in eliciting ABTAA-mediated amelioration of sepsis mortality.

To assess the physiological benefits of ABTAA treatment in sepsis, the authors performed microcomputed tomography on the vital organs of the model mice. The antibody reduced lung oedema by $60 \%$ compared with vehicle-treated mice, as well as significantly preserving lung parenchymal integrity and suppressing pericyte loss. Furthermore, ABTAA substantially protected renal peritubular capillaries and preserved the depth of the endothelial glycocalyx (an important barrier that prevents adhesion of blood cells to the vessel endothelium).

Last, ABTAA treatment was associated with reduced neutrophil recruitment to the lung and blunted serum levels of the inflammatory molecules tumour necrosis factor and interleukin-6.

This study presents a novel mechanism of action of a therapeutic antibody that harnesses beneficial TIE2 signalling and could help to address the unmet medical need in sepsis and other vascular diseases.

Katie Kingwell

ORIGINAL ARTICLE Han, S. et al. Amelioration of sepsis by TIE2 activation-induced vascular protection. Sci. Transl. Med. 8, 335ra55 (2016) FURTHER READING Fink, M. P. \& Warren, H. S. Strategies to improve drug development for sepsis. Nat. Rev. Drug Discov. 13, 741-758 (2014) 